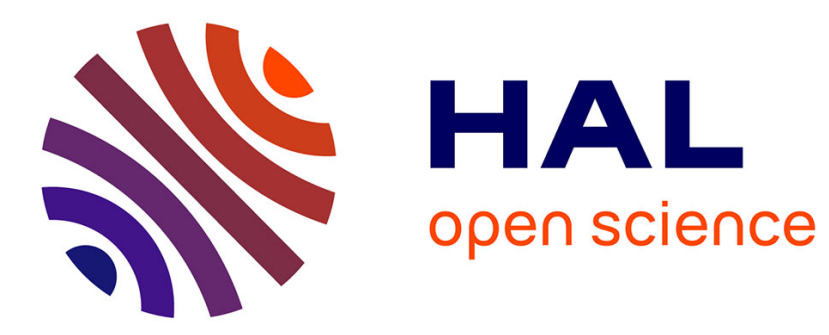

\title{
Root growth and force chains in a granular soil
}

Mahmoud Fakih, Jean-Yves Delenne, Farhang Radjai, Thierry Fourcaud

\section{To cite this version:}

Mahmoud Fakih, Jean-Yves Delenne, Farhang Radjai, Thierry Fourcaud. Root growth and force chains in a granular soil. Physical Review E , 2019, 99 (4), 10.1103/PhysRevE.99.042903 . hal-02137110

\section{HAL Id: hal-02137110 \\ https://hal.umontpellier.fr/hal-02137110}

Submitted on 27 May 2019

HAL is a multi-disciplinary open access archive for the deposit and dissemination of scientific research documents, whether they are published or not. The documents may come from teaching and research institutions in France or abroad, or from public or private research centers.
L'archive ouverte pluridisciplinaire HAL, est destinée au dépôt et à la diffusion de documents scientifiques de niveau recherche, publiés ou non, émanant des établissements d'enseignement et de recherche français ou étrangers, des laboratoires publics ou privés. 


\title{
Root growth and force chains in a granular soil
}

\author{
Mahmoud Fakih,,$^{1,2, *}$ Jean-Yves Delenne, ${ }^{3, \dagger}$ Farhang Radjai, ${ }^{1,4, \$}$ and Thierry Fourcaud ${ }^{2, \S}$ \\ ${ }^{1}$ LMGC, Université de Montpellier, CNRS, 163 rue Auguste Broussonnet, 34095 Montpellier, France \\ ${ }^{2}$ AMAP, CIRAD, CNRS, INRA, IRD, University of Montpellier, TA A51/PS2, 34398 Montpellier, France \\ ${ }^{3}$ IATE, INRA, CIRAD, SupAgro, University of Montpellier, 2 place Pierre Viala, 34060 Montpellier, France \\ ${ }^{4}\langle M S E\rangle^{2}$, UMI 3466 CNRS-MIT, Massachusetts Institute of Technology, 77 Massachusetts Avenue, Cambridge 02139, USA
}

(Received 22 November 2018; published 15 April 2019)

\begin{abstract}
Roots provide basic functions to plants such as water and nutrient uptake and anchoring in soil. The growth and development of root systems contribute to colonizing the surrounding soil and optimizing the access to resources. It is generally known that the variability of plant root architecture results from the combination of genetic, physiological, and environmental factors, in particular soil mechanical resistance. However, this last factor has never been investigated at the soil grain scale for roots. In this paper, we are interested in the effect of the disordered texture of granular soils on the evolution of forces experienced by the root cap during its growth. We introduce a numerical model in which the root is modeled as a flexible self-elongating tube that probes a soil composed of solid particles. By means of extensive simulations, we show that the forces exerted on the root cap reflect interparticle force chains. Our simulations also show that the mean force declines exponentially with root flexibility, the highest force corresponding to the soil hardness. Furthermore, we find that this functional dependence is characterized by a single dimensionless parameter that combines granular structure and root bending stiffness. This finding will be useful to further address the biological issues of mechanosensing and thigmomorphogenesis in plant roots.
\end{abstract}

DOI: 10.1103/PhysRevE.99.042903

\section{INTRODUCTION}

The plant-root system displays two main functions essential for plant growth, which are mechanical anchoring and water and nutrient uptake [1,2]. Root growth and development, including branching, allow the plant to explore and colonize the surrounding soil and optimizing the access to resources. These processes are driven by genetic, biotic, and abiotic factors. The observed variability of root architecture, defined as the evolution of the root system geometry and topology, can consequently result from the combination of endogenous instabilities [3] and environmental heterogeneities. Gravitropism, i.e., the growth response of plants to gravity, is also an important aspect of a root's design [4]. The local effect of water and nutrient content on root growth and branching has been investigated by several authors [5-8]. Some of them used numerical process-based models to analyze and quantify water flows within a root-soil domain at the root and plant scales [9-11].

It is well known that the soil mechanical impedance (in the sense of resistance to penetration) is also an important factor that can affect root growth and hence crop productivity [12]. Experimental studies have been carried out in order to characterize and quantify this mechanical impact in different

\footnotetext{
*Presently at Department of Civil Engineering, Lebanese American University, Beirut, Lebanon; mahmoud.fakih@lau.edu.lb

†jean-yves.delenne@umontpellier.fr

†franck.radjai@umontpellier.fr

\$thierry.fourcaud@cirad.fr
}

soil types at the level of a single root [13-15] or at the plant scale $[16,17]$, sometimes considering the combined effects of soil strength and water content on the development and anchorage of roots $[18,19]$. Some of these studies [20,21] were supported by numerical simulations using the finite-element method where the soil was simplified as a continuous domain [22]. Such simulation approaches are, however, not able to capture grain-scale effects that can have significant effects on root growth trajectory and root physiological responses. The integration of such small-scale phenomena at the level of the whole root system can partly explain their architectural variability.

Representing the complex interactions between a single growing root and soil deformation requires a grain-scale approach where the inhomogeneous texture of the soil can be explicitly described. The packing fraction (volume fraction of particles) and interstitial pore distribution may influence the root growth via their effects on force transmission, soil strength, aeration, and hydraulic permeability $[5,6,23,24]$. The packing texture determines the maximum pressure exerted on the root [25]. This value should be compared with threshold pressures associated to particular physiological responses, e.g., trajectory deviation due to an active movement of the root (tropism) or full arrest of the root elongation [25-28].

In the same way, the frictional resistance to sliding increases the overall resistance of the soil to penetration, making the root-soil friction angle an important parameter [20]. Conversely, depending on the root flexibility and diameter, the growth of a root inside a granular soil tends to modify the soil texture. Bengough et al. [29] studied the effect of a growing root on the particle displacements within a sandbox 
by means of the particle image velocimetry technique. They found that the displacements decrease with distance from the root over a long distance. Hence, the presence of growing roots or dead roots inside a soil affects the growth conditions of each root via their footprint on the packing fraction and pore distributions.

The force that a root must exert to penetrate the soil can be measured at the radicle initiation by measuring the force exerted on the seed $[30,31]$. Once the root has penetrated a distance about 3 times its diameter into soil, it gets anchored and therefore the force transmitted back from the tip along the root to the seed is no longer equal to the force required to penetrate the soil $[32,33]$. Attempts to measure the force acting at the root tip have been made in the laboratory in order to evaluate the maximum growth pressure for different root species $[28,34,35]$. The radial growth pressure has also been investigated by means of photoelastic grains [14]. However, such measurements are difficult or even impossible to perform in larger-scale experiments or natural conditions.

At the grain scale, the root cap interacts with the highly inhomogeneous texture of the soil. A flexible and narrow root may easily explore the pore space without being able to dislodge the grains equilibrated by the normal and frictional forces exerted by their neighboring grains. But there is always a large number of grains that are subjected to weak forces and belong to what has come to be known as the "weak force network" [36,37]. As the root rigidity increases, the force necessary to bend a root during its growth may overcome large interparticle forces and the strong force chains observed in granular materials [38-42]. Hence, for understanding the effect of soil inhomogeneity on the root growth and architecture, a key issue is to determine how the interparticle forces and force chains are reflected in the forces experienced by the root cap.

In this paper, we investigate the distribution and evolution of forces exerted by soil grains on a root modeled as a self-elongating elastic tube of constant thickness. Active apex movements such as gravitropism or other deviations due to anisotropic cell growth are not considered here, i.e., the root path is only driven by external forces exerted by the surrounding grains. This choice was made in order to dissociate the external mechanical component from the biological component of root trajectory. The modeling framework is the discrete element method (DEM) in which the soil is represented by a granular material composed of rigid grains [43-46]. The root is characterized by its longitudinal, tangential, and bending stiffnesses, as well as its diameter and growth rate. We consider different values of the root bending stiffness and granular samples of different values of packing fraction and particle size distribution. As we shall see, the root-particle interaction force has a well-defined probability density, and the mean force normalized by the hardness of the granular material is governed by a robust scaling as a function of the root bending stiffness.

In the following, we first describe the root model and numerical procedures in Sec. II. In Sec. III, we analyze the probability density function of the forces exerted on the root cap. Section IV presents the evolution of the mean force with root flexibility. Then, in Sec. V, we consider the effects of soil properties such as packing fraction and cohesive stresses on the mean force. We conclude in Sec. VI with a summary of the main findings of this work and a brief discussion of its possible extensions.

\section{NUMERICAL MODEL AND PROCEDURES}

The general modeling framework for particle dynamics applied to a collection of solid particles is the DEM $[43,44]$. In this method, the particle motions are computed by stepwise integration of the equations of motion for all particles by accounting for their frictional contact interactions and the gravity and boundary forces. We use the same framework to model the root, defined as a flexible tube represented by an array of segments interconnected by linear springs and growing as a result of its continuous elongation. The general numerical model and simulations are in two dimensions as the root growth requires long-time simulations and we need to perform a large number of independent simulations in order to be able to assess the variability of the results and the effect of system parameters. We present below in more detail the particle interactions and our root model.

The soil particles are two-dimensional (2D) disks interacting via a reaction force $\vec{f}$ defined by its two components $f_{n}$ and $f_{t}$ along and perpendicular to the contact normal $\vec{n}$, respectively:

$$
f=f_{n} \vec{n}+f_{t} \vec{t}
$$

where $\vec{t}$ is the unit vector perpendicular to $\vec{n}$. The normal force is the sum of three different forces:

$$
f_{n}=f_{n}^{e}+f_{n}^{d}+f_{n}^{c},
$$

where $f_{n}^{e}$ is the repulsive elastic force, $f_{n}^{d}$ is a viscous damping force, and $f_{n}^{c}$ is a cohesion force induced by solid or liquid bonding between the particles. The elastic normal force is assumed to be a function of the contact deflection $\delta_{n}$, assumed to be equal to the overlap between the disks for small contact deformations. We use a linear elastic law as far as there is an overlap between the particles, whereas the force vanishes when there is no contact:

$$
f_{n}^{e}=\left\{\begin{array}{ll}
-K_{n} \delta_{n} & \text { if } \delta_{n}<0 \\
0 & \text { otherwise }
\end{array},\right.
$$

where $K_{n}$ is the contact stiffness.

In the DEM particle dynamics simulations, it is necessary to introduce also viscous damping to account for energy dissipation due to inelastic collisions between particles. To make the energy restitution coefficient independent of the particle mass, the damping force is assumed to depend also on the spring stiffness and particle mass as follows:

$$
f_{n}^{d}= \begin{cases}-2 \alpha_{n} \sqrt{m K_{n}} \dot{\delta}_{n} & \text { if } \dot{\delta}_{n}<0 \\ 0 & \text { otherwise }\end{cases}
$$

where $m=m_{i} m_{j} /\left(m_{i}+m_{j}\right)$ is the reduced mass of the two touching particles $i$ and $j$ and $\alpha_{n}$ is the damping constant varying between 0 and 1 . The cohesion force $f_{n}^{c}$ can have different expressions depending on the source of cohesion [47]. 


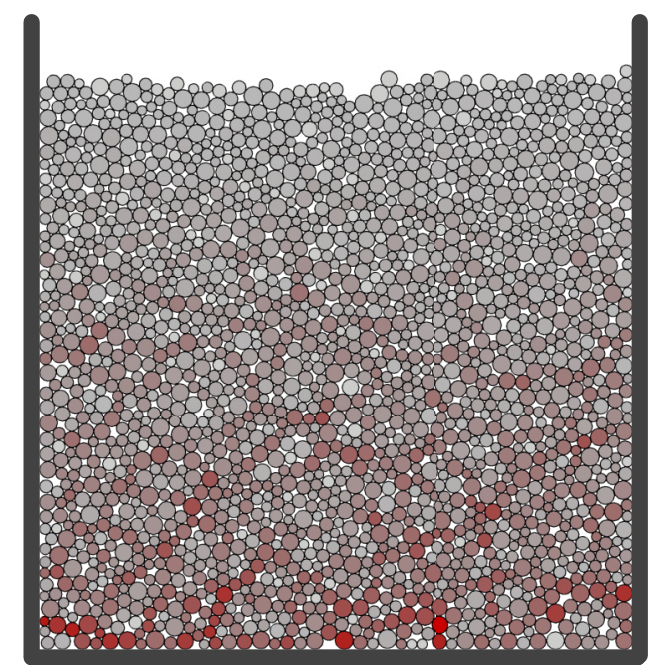

FIG. 1. A granular sample at the end of relaxation to equilibrium. Color intensity is proportional to average normal force supported by each particle.

For the friction force $f_{t}$, we use an elastic-regularized Coulomb law $[48,49]$ :

$$
f_{t}=\left\{\begin{array}{ll}
-\operatorname{sgn}\left(\dot{\delta}_{t}\right) \mu f_{n} & \text { if }\left|\hat{f}_{t}\right| \geqslant \mu f_{n} \\
K_{t} \delta_{t}+\gamma_{t} \dot{\delta}_{t} & \text { otherwise }
\end{array},\right.
$$

where $K_{t}$ is the stiffness of the tangential spring, $\gamma_{t}$ is the tangential damping constant, $\delta_{t}$ is tangential spring elongation since the contact between two particles was established, $\dot{\delta}_{t}$ is relative tangential velocity, and $\mu$ is the friction coefficient.

The granular samples are prepared by sedimentation: The particles are placed on the nodes of a triangular lattice without overlap and allowed to fall into a box under the action their own weights. The final state after sufficient relaxation of the particles is a dense packing in which all forces on all particles are balanced. A weak size polydispersity of particles is introduced to avoid long-range ordering of the disks in 2D. The particle radii $R_{p}$ are distributed between $R_{\min }=$ $1 \mathrm{~mm}$ and $R_{\max }=2 \mathrm{~mm}$ with a uniform distribution of their volumes. This distribution ensures that the total volumes of the particles in all particle size classes are equal, and it leads to dense packings [50]. The total number $N$ of particles is 1500. An example of a sample used for simulations with a representation of particle pressures is displayed in Fig. 1.

The root is modeled as a self-elongating spheroline that can change its direction under the action of the forces exerted by soil particles. A spheroline is the locus of all points at a given distance from a segment and shrinks to a sphere when the length of the segment tends to zero. It can also be described as a rounded-cap rectangle. We begin with a seed defined by an immobile disk of radius $R_{r}$ placed on top of the sample. The initial direction of growth $\alpha_{1}$ is assumed to be vertical. We make the root grow from this seed by replacing the disk by a spheroline of radius $R_{r}$ defined by a segment $A_{1} A$, where $A$ is a new point added vertically below $A_{1}$; see Fig. 2. The root keeps growing by incremental displacement of the point $A$ at a constant rate $u_{g}$. As long as the growth distance $A_{1} A$ is below a maximum length $\ell_{g}$, the spheroline

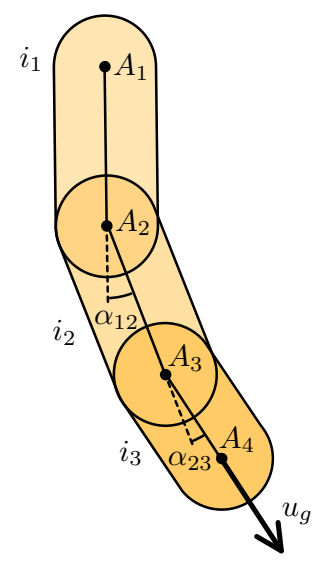

FIG. 2. Root particle geometry and connection between consecutive root elements.

keeps its direction $\alpha_{1}$. When $A_{1} A=\ell_{g}$, the end point $A \equiv A_{2}$ of the spheroline becomes the seed for the growth of a new spheroline in a new direction $\alpha_{2}$ determined by the forces acting on it; see Fig. 2. The same process is repeated with the new spheroline until the length of the new root element reaches $\ell_{g}$. By iterating this process, we obtain a sequence of points $A_{1} A_{2} A_{3} \ldots$ along successive directions $\alpha_{1} \alpha_{2} \alpha_{3} \ldots$ which describes the geometry of a root of thickness $d_{\text {root }}=$ $2 R_{r}$ grown from the seed $A_{1}$. Figure 3 shows an example of a root obtained by this procedure. The ratio $\ell_{g} / R_{\min }$ controls the precision with which the shape of the root is defined. In the following, we refer to the spherolines as "root elements."

The flexibility of the root depends on the bending (or torsional) resistance between consecutive root elements. The endpoint of each root element $i$ is connected to the initial point of the next root element $i+1$ by two linear springs. The elongational spring connecting the two points exerts a force $\vec{f}_{i(i+1)}$ proportional to their separation distance with a longitudinal stiffness $K_{e}$. In the absence of all other forces, $\vec{f}_{i(i+1)}$ vanishes when the two points coincide. The stiffness $K_{e}$ defines the axial stiffness of the root. The two elements

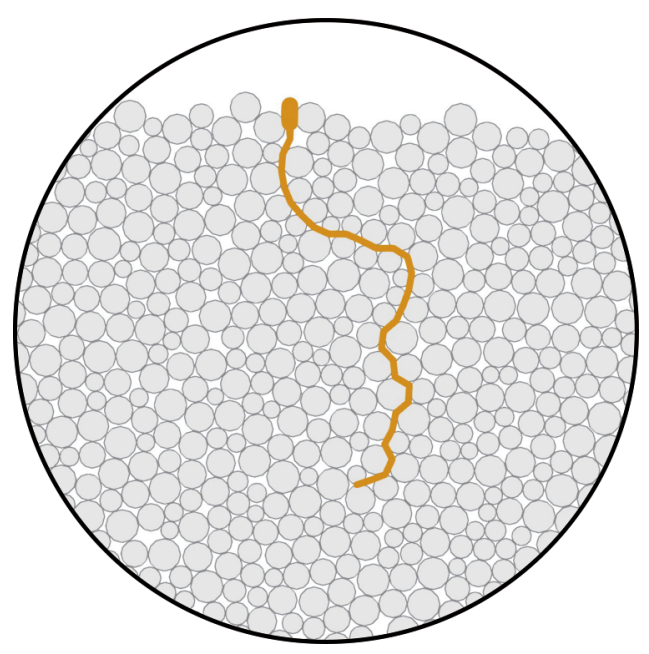

FIG. 3. A root grown from a seed placed at the top of a granular sample. 
are also connected by a linear torsional spring with a torque $M_{i(i+1)}$ proportional to the difference $\alpha_{i}-\alpha_{i+1}$ between their directions:

$$
M_{i(i+1)}=K_{b} \alpha_{i(i+1)}=K_{b}\left(\alpha_{i}-\alpha_{i+1}\right),
$$

where $K_{b}$ is the bending stiffness of the root.

The motion of the center of mass $\vec{r}_{i}$ of each root element or soil particle $i$ along the $x$ and $y$ directions is governed by the equations of motion:

$$
m_{i} \ddot{\vec{r}}_{i}=\sum_{j \in \Omega^{i}} \vec{f}_{i j}
$$

where $m_{i}$ is the mass and the summation runs over the set $\Omega^{i}$ of all neighboring particles or root elements $j$ exerting a force $\overrightarrow{f_{i j}}$ on the particle or root element $i$. The angular motion of each root element $i$ is governed by the moment equation

$$
I_{i} \ddot{\alpha}_{i}=M_{i(i-1)}+M_{i(i+1)}+\sum_{j \in \Omega^{i}} \vec{c}_{i j} \times \vec{f}_{i j},
$$

where $I_{i}$ is the moment of inertia of the root element with respect to its center of mass, the torques $M_{i(i-1)}$ and $M_{i(i+1)}$ are given by Eq. (6), and $\vec{c}_{i j}$ is the contact vector joining the center of mass of element $i$ to its contact point with particle or element $j$. The angular motions of the particles are governed by the same equation without the torque terms.

The above model of a "discrete" growing root implies that a root emanating from a seed will grow along a straight vertical line in the absence of soil particles and gravity. In the presence of soil particles, the root will frequently meet the particles on which it can exert a force, leading either to the displacement of the particles or elastic deformation of the root itself. Because of elasticity, however, the root resumes its reference shape as a straight line if the soil particles are removed. This is not what one observes in real roots, which, unless damaged, keep practically their shape when removed from a soil. This is due to the remodeling of the root during its growth as a result of the accretion of new wood material at the surface and/or a change in material internal properties [51] and leads to two different effects: (1) stiffening of the root and (2) transformation of elastic reorientations of the root into permanent deflections. To account for these evolutions of the root, we assume that the bending stiffness $K_{b}$ between the last growing root element, which represents the meristem, and the element to which it is attached is much lower than the stiffness $K_{b}^{\prime}$ of the preceding elements. In this way, the tip of the root can more easily probe the pore space between soil particles than the upstream parts of the root.

Moreover, we allow the reference equilibrium angle $\alpha^{0} i(i+1)$ between consecutive root elements to evolve. The initial reference angle is zero according to Eq. (6), corresponding to a zero torque when the two root elements are colinear. As the root grows, at the same time as a new root element is created, we set all reference angles between all root elements, except for that between the new element and its preceding element, to their current values. As a result, the elastic torque acting between all those elements is reset to zero, and the torque in the following steps of computation is given by

$$
M_{i j}=K_{b}^{\prime}\left(\alpha_{i j}-\alpha_{i j}^{0}\right) .
$$

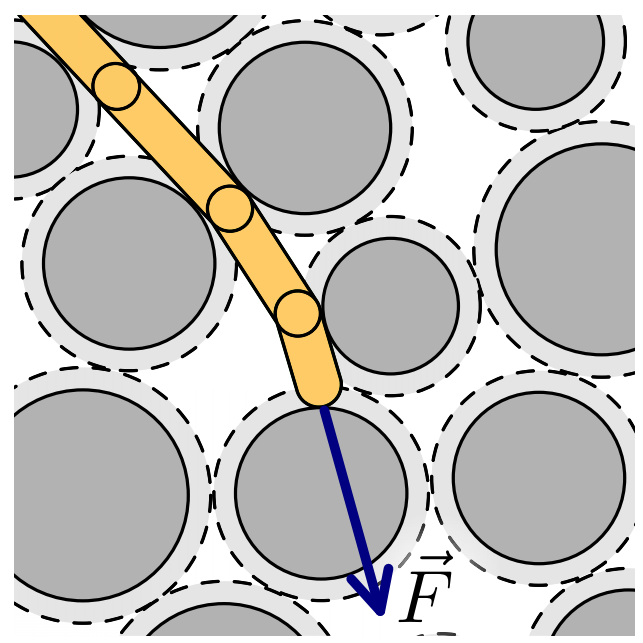

FIG. 4. A growing root and the interparticle gaps seen by the root.

It should be noted that, although the reorientations of the meristem are much easier than other parts of the root, this evolution of parameters does not prevent later variations of the angles as they still can change by elastic deflections depending on the forces exerted by the soil particles.

Another aspect that needs to be considered in 2D simulations is that, in contrast to 3D packings, all pores in a dense 2D packing are closed, implying that the root cannot grow without dislodging the particles. In order to relax this 2D pathology, we use two different radii for the particles: one radius $R_{p}$ for particle-particle contacts and a slightly smaller radius $R_{p}^{\prime}=$ $R_{p}-0.5 d_{\text {gap }}$ for the particle-root contacts. Hence, when two particles are in contact, the root "sees" a gap of width $d_{\text {gap }}$ between the two particles through which it can pass without touching the particles if the root diameter $d_{\text {root }}$ is smaller than $d_{\text {gap }}$, as shown in Fig. 4 . The ratio $s=d_{\text {root }} / d_{\text {gap }}$ is a model parameter that plays the same role as the ratio of the root diameter to the pore size in 3D and, as we shall see, it will influence the force required for the root to dislodge the particles.

As to the friction coefficients between root elements and particles, we consider the plausible assumption that the friction coefficient of soil particles with the meristem is smaller than with other root elements because the sloughing of cells from the root cap produces a low-friction disposable sleeve [52]. In the simulations reported in this paper, we set the friction coefficient between the root cap and particle to zero. The friction coefficient between particles and with other root elements was set to $\mu=0.4$.

We used the velocity-Verlet stepping scheme to solve the equations of motion for all particles and root elements [53]. The solver was also optimized by using the cell method for the book-keeping of close neighbors [54]. We set $K_{n}=K_{t}=$ $10^{-6} \mathrm{~N} / \mathrm{m}$ and performed a large number of simulations with different values of bending stiffness $K_{b}$, elementary growth length $\ell_{g}$, particle sizes $R_{\min }$ and $R_{\max }$, gap parameter $s$, and packing fraction $\Phi$ of the soil. With each set of parameter values, we performed five independent simulations by changing the position of the seed on top of the sample in order to evaluate the variability due to granular disorder. 


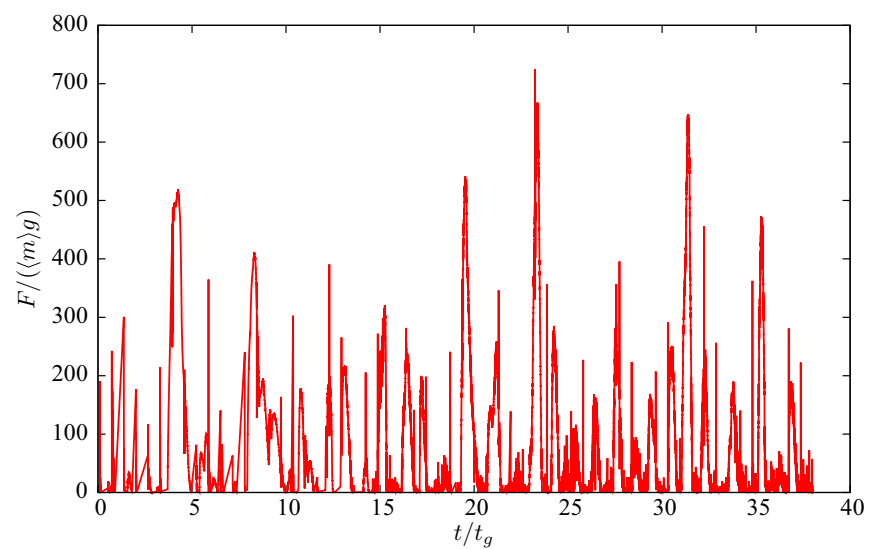

FIG. 5. Evolution of the force $F$ experienced by the root cap during its growth as a function of normalized time. The force is normalized by the mean particle weight.

In all simulations, the growth rate $u_{g}$ was set to a small value in order to avoid inertial effects that are basically absent from the very slow growth process of roots. The typical growth time $\tau$ is the time necessary for the root tip to grow over a distance of the order of an average soil particle diameter:

$$
\tau=\frac{\langle d\rangle}{u_{g}} .
$$

This time should be compared with the time necessary for a particle dislodged from its equilibrium state to relax back to equilibrium. The typical distance being of the order of one particle diameter, the characteristic relaxation time is

$$
t_{g}=\left(\frac{\langle d\rangle}{g}\right)^{1 / 2}
$$

where $g$ is the gravity. Hence, for a quasistatic growth and high time resolution we imposed $\delta t \ll t_{g} \ll \tau$, where $\delta t$ is the simulation time step.

\section{ROOT-PARTICLE FORCE DISTRIBUTIONS}

During the root growth, the positions $x$ and $y$ of the root tip define its trajectory. We consider here the total force exerted by the soil particles on the root cap, i.e., the force acting on the growing root element. Its projection along this root element is the "axial force" $F$ that resists root growth; see Fig. 4. Figure 5 displays the evolution of $F$ with time for a root of bending stiffness $K_{b}=1 \mathrm{Nm}$ and $s=1.54$ in a dense sample of 1500 particles. The force is normalized by the mean weight $\langle m\rangle g$ of a single particle, whereas the time is normalized by the growth time $t_{g}$. We see that the force signal undergoes large variations between zero and values as high as 700 times the mean particle weight nearly independently of its depth inside the soil. The zero force values correspond to the passage of the root in the pores without contact with particles, whereas high values occur when the root meets the particles balanced by strong force chains. The strong forces in granular materials can be much larger than the mean force [36]. Although the mean force increases with depth, this increase is negligible
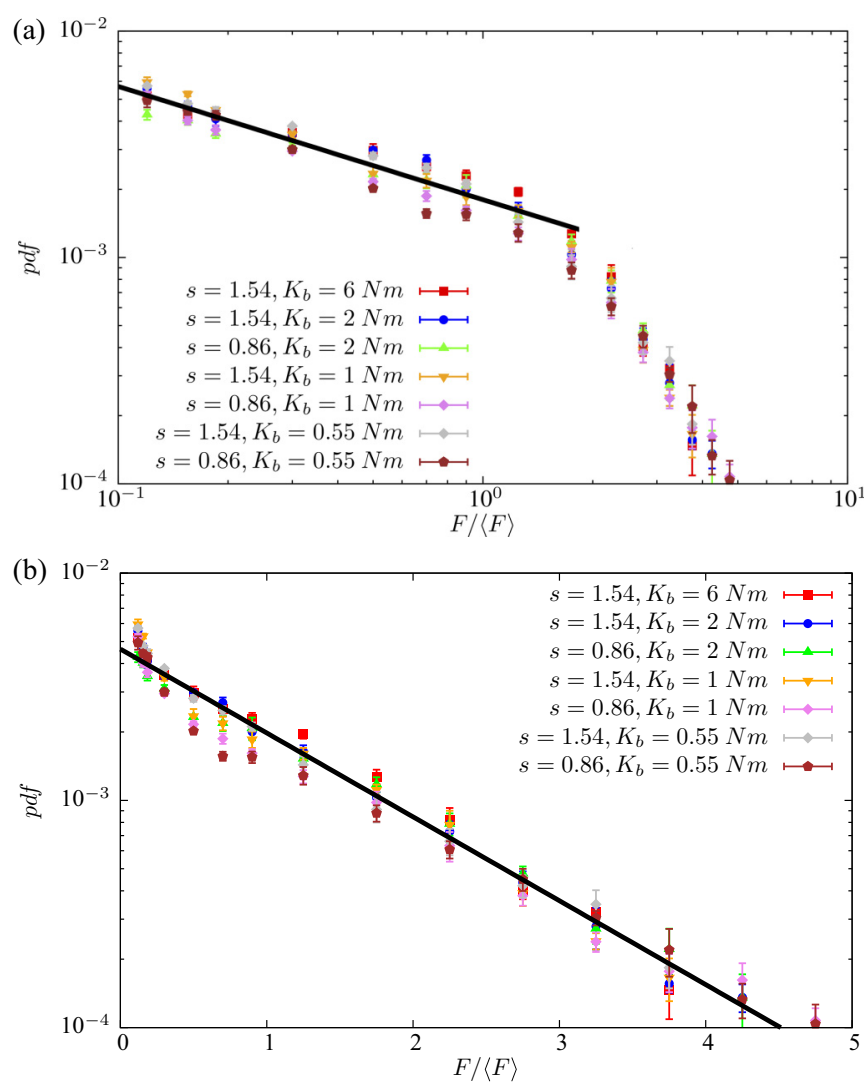

FIG. 6. Probability density function of forces experienced by a growing root in a granular packing in log-log and log-linear scales for different values of the gap parameter $s$ and bending stiffness $K_{b}$ of the root.

compared to the large force fluctuations arising from rootparticle interactions.

Figure 6 shows the probability density function (pdf) of root-particle forces on log-log and log-linear scales for two different values of $s$ (above and below 1) and for a set of values of $K_{b}$ ranging from 0.55 to $6 \mathrm{Nm}$. We see that, despite the broad range of the values of $K_{b}$, all the data points collapse nicely on the same curve when the forces are normalized by the mean force $\langle F\rangle$. The small deviations reflect the packing disorder and finite system size. The distribution of forces below the mean force (weak forces) is a power law $F^{-\alpha}$ with exponent $\alpha \simeq 0.5$ over one decade. The distribution of forces above the mean force (strong forces) is well fitted by an exponential falloff $e^{-\beta F /\langle F\rangle}$ with parameter $\beta \simeq 0.85$.

This distribution with its transition from a decreasing power-law behavior to an exponential falloff around the mean force is reminiscent of the pdf of contact forces in granular materials $[37-39,55]$. This "bimodal" distribution can be approximated by $[40,41,56]$

$$
P(F)= \begin{cases}k\left(\frac{F}{\langle F\rangle}\right)^{-\alpha} & N /\langle F\rangle<1 \\ k e^{\beta(1-F /\langle F\rangle)} & F /\langle F\rangle>1\end{cases}
$$

where $k$ is the normalization factor given by

$$
\frac{1}{k}=\frac{1}{1-\alpha}+\frac{1}{\beta} \text {. }
$$


Moreover, considering the mean force $\langle F\rangle$ as the point of crossover between the two parts of the distribution, we get the following relation between the exponents:

$$
\beta^{2}=(1-\alpha)(2-\alpha)
$$

This relation is in excellent agreement with the values of the exponents $\alpha$ and $\beta$ measured from Fig. 5. Although the values of these exponents for contact force distributions in granular materials are different (lower values of $\alpha$ and larger values of $\beta$ depending on the soil properties such as size polydispersity [40]), the similarity between their pdf's is a strong indication that a growing root probes the weak and strong force networks. Their independence from the root bending stiffness is consistent with this assumption.

\section{EFFECT OF THE ROOT BENDING STIFFNESS}

Although the force pdf is independent of the root and soil characteristics, the mean force $\langle F\rangle$ exerted on the root cap during its growth is sensitive to the root bending stiffness $K_{b}$ and soil parameters such as packing fraction $\Phi$, mean particle diameter $\langle d\rangle$, and cohesive forces $f_{c}$ between particles. Herein we consider the effect of $K_{b}$ as a major root parameter that controls the ability of the root to change its direction and explore the pore space. With low bending stiffness the root is more flexible and tends to follow the tortuous space of the pores and gaps between particles, whereas for large bending stiffness the root will be able to dislodge the particles and will therefore follow a path close to a straight line.

Figure 7 shows $\langle F\rangle$ as a function of $K_{b}$ for $s=0.86$. We see that the mean force increases from a weak value $F_{0}$ at very low values of $K_{b}$ and levels off to a constant value $H$ at large values of $K_{b}$. For each value of $K_{b}$, the data point represents the average force obtained from five independent simulations in which only the position of the seed is changed on top of the sample. The error bars represent the corresponding values of the standard deviation. Within statistical precision, the evolution of $\langle F\rangle$ with $K_{b}$ is well fitted by an exponential

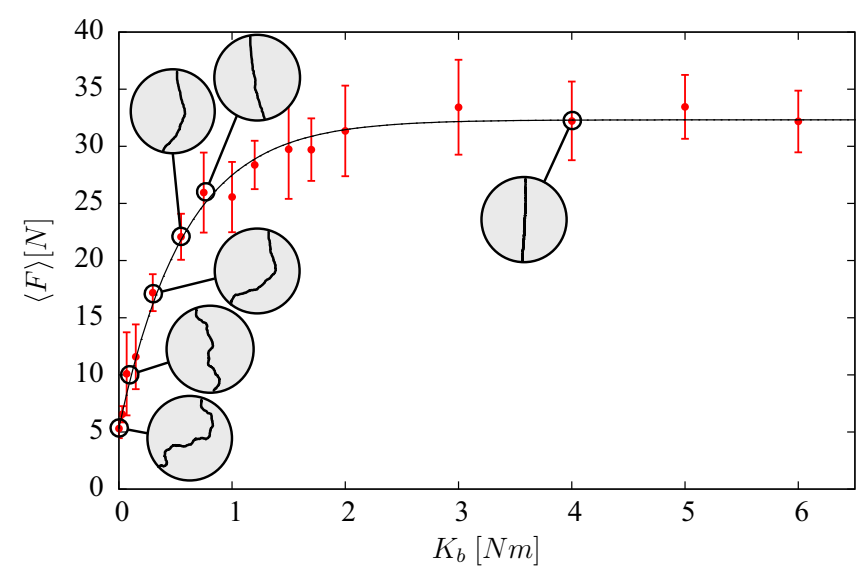

FIG. 7. The mean force $\langle F\rangle$ experienced by the root cap as a function of bending stiffness $K_{b}$ for $s=0.86$. The error bars represent standard deviation for five independent simulations. The circular insets display a portion of the root for several values of $K_{b}$. function

$$
\langle F\rangle=F_{0}+\left(H-F_{0}\right)\left(1-e^{-a^{\prime} K_{b}}\right),
$$

where the parameter $a^{\prime}$ has the dimension $N^{-1} m^{-1}$. In the two limits of very high and low values of $K_{b}$, the force is independent of $H$. At high values, $\langle F\rangle=H$ coincides with the resistance of the granular medium to the penetration of a rigid bar, whereas at low values the force $\langle F\rangle=F_{0}$ results from the collisions of the root with the soil particles and its reorientations are not prohibited by the root bending stiffness. As we shall see below, we find $F_{0} \simeq 0.1 H$. In Fig. 7 we also have displayed a portion of the root at different steps of its growth with a shape that evolves gradually from a random walk in the pore space to a straight line as $K_{b}$ increases.

Equation (15) is interesting as it describes in a simple and quantitative way the effect of root flexibility on the mean force involved in root growth. This relation makes it possible to predict the mean force exerted on the root cap during it growth from the knowledge of the root stiffness and the soil hardness $H$ defined as the resistance of the soil to the penetration of a rigid bar as in penetrometer experiments [57,58]. Equation (15) shows clearly that the mean force experienced by the root cap can actually be much lower than $H$ as measured from the ground by a penetrometer depending on the root flexibility. It should be noted here that the longitudinal and tangential stiffnesses of the root are set to a high value, and we checked that their values are not significant for the results presented below.

Equation (15) with its values of $H$ and $F_{0}$ was obtained for specific values of soil parameters such as particle size distribution, gap size, and packing fraction without cohesive forces acting between soil particles. In order to examine the robustness of Eq. (15) under more general conditions and investigate the influence of relevant parameters, we performed extensive simulations of root growth with a range of the parameter values.

\section{INFLUENCE OF SOIL PARAMETERS}

We first consider here the effect of the packing fraction $\Phi$, which is an important soil property that depends on material parameters such as friction coefficient $\mu$ between particles but also on the history of soil deformations. As we would like to single out the effect of the packing fraction, we fix all material parameters but apply a preparation method to obtain different values of $\Phi$.

To prepare samples with different values of the packing fraction, we first prepare a dense packing of 2000 particles by setting $\mu$ to zero and allowing the particles fall into the simulation box from their initially random positions in space. The zero value of $\mu$ reduces the probability of arching and leads to a dense packing with $\Phi=0.835$. Then we set $\mu=$ 0.4 and remove one particle at a time randomly selected from the packing. After each removal, we allow the packing to relax toward equilibrium over several time steps. The equilibrium criterion is chosen according to the particle velocities and in order to make the process numerically efficient. Thus, a new particle is removed when the mean particle velocity in the sample is much lower than $\sqrt{g\langle d\rangle}$, which is the falling time of a particle of average diameter $\langle d\rangle$ over a distance equal to its 


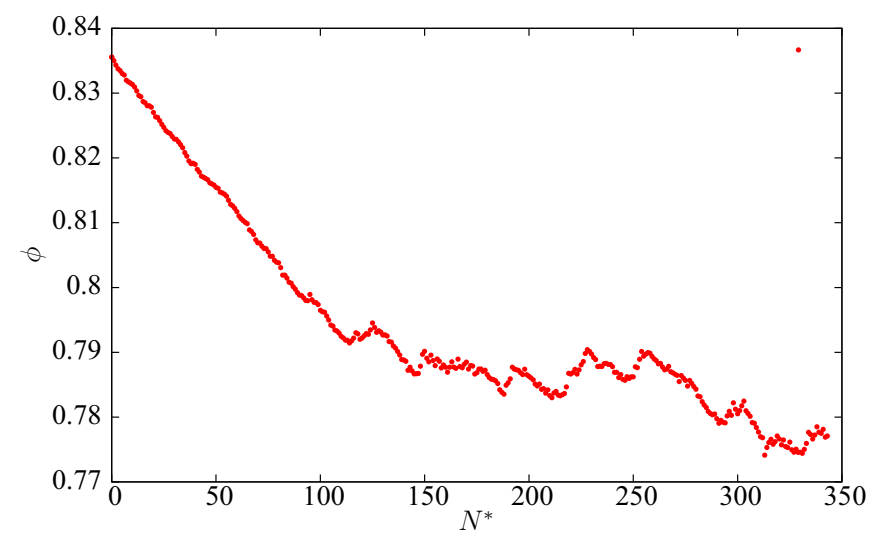

FIG. 8. Variation of packing fraction $\Phi$ as a function of the number $N^{*}$ of particles removed.

diameter. Removing a particle creates a local imbalance and therefore entails a rearrangement of the particles. But this rearrangement is not necessarily local. As a result, $\Phi$ decreases by removal but increases again by relaxation. Since we begin with high packing fraction, the removal-relaxation process leads to a gradual decrease of $\Phi$ until a statistical balance is achieved between the phases of decrease and increase of $\Phi$, as shown in Fig. 8. The average packing fraction in this steady state is $\simeq 0.78$ after the removal of 350 particles.

We selected three samples of packing fractions $0.77,0.8$, and 0.83 , displayed in Fig. 9. Despite locally large variations of porosity, we checked that there is no porosity gradient by dividing each sample to eight horizontal layers. Then we carried out root growth simulations for increasing values of $K_{b}$ in each sample. Figure 10(a) shows the mean force as a function of $K_{b}$ for the three samples. The asymptotic force $H$ increases considerably with $\Phi$. But, within statistical precision, the data are well fit by the exponential form of Eq. (15), and thus for each value of $K_{b}$ the mean force increases with packing fraction.

The exponential fit of Eq. (15) clearly suggests that the mean force should be scaled by $H$ but this normalization of the forces does not ensure that they will collapse on the same curve as the values of the prefactor $a^{\prime}$ in the exponent are different in the three exponential fits. Hence, we also need
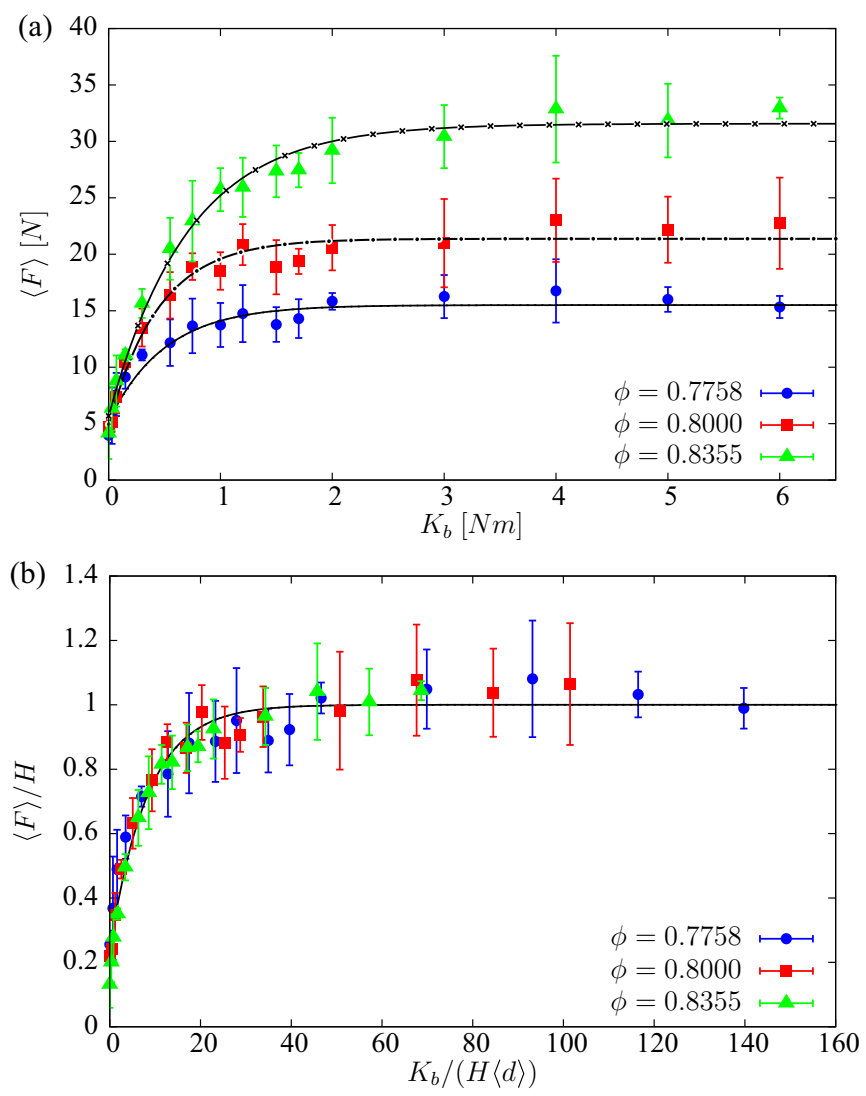

FIG. 10. (a) The mean force as a function of the root bending stiffness for three samples of different packing fractions. (b) The same data points with the mean force normalized by soil hardness $H$ and bending stiffness normalized by $H$ times the mean particle size $\langle d\rangle$. The lines are exponential fits according to Eq. (15) in (a) and Eq. (16) in (b).

to scale $K_{b}$ by an appropriate material parameter. $K_{b}$ has the dimension of a force multiplied by a length. $\ell_{g}$ being a model discretization parameter, the only characteristic length of the packing is the average particle size $\langle d\rangle$. We also have two characteristic forces: (1) the mean particle weight $\langle m\rangle g$ and (2) the hardness $H$. As previously discussed, the root-particle forces are considerably larger than $\langle m\rangle g$ and they result from
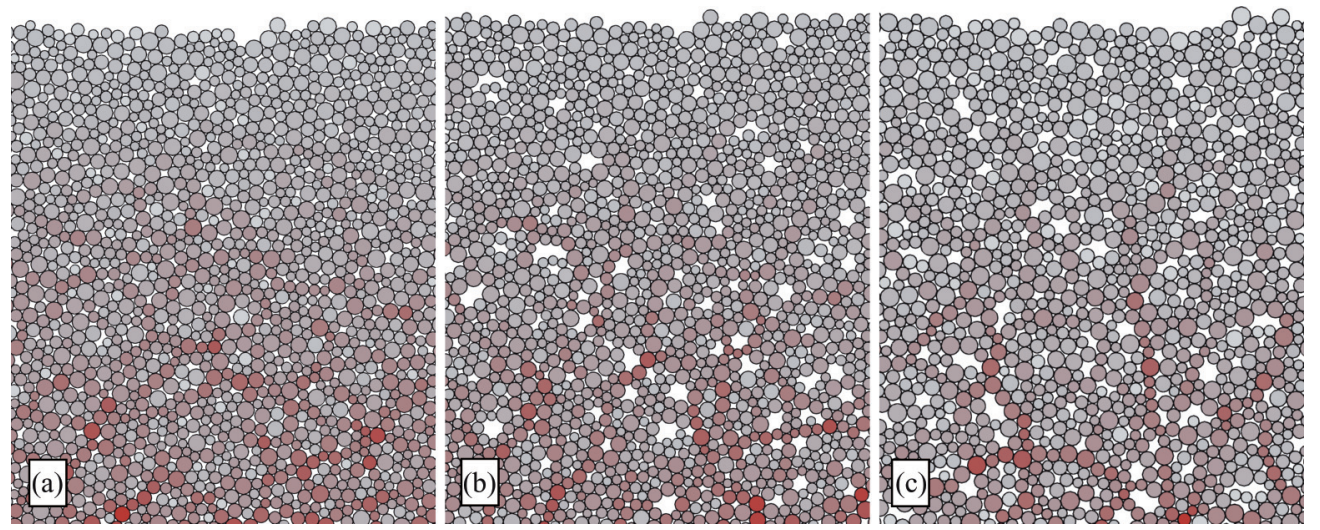

FIG. 9. Samples of different packing fractions obtained by a removal-relaxation process. Line thickness is proportional to normal force between particles. 
both the interparticle force chains and the root-particle interactions. The only force reflecting both these aspects is $H$. This suggests that we should normalize the bending stiffness by $H\langle d\rangle$. As observed in Fig. 10(b), with this scaling all the data points from the three samples nicely collapse on a single curve well fit by

$$
\frac{\langle F\rangle}{H}=1-\left(1-\frac{F_{0}}{H}\right) e^{-a K_{b} /(H\langle d\rangle)},
$$

where $a \simeq 0.15$ and $F_{0} / H \simeq 0.1$. This scaling means that the effect of the packing fraction $\Phi$ is fully captured by the dependence of $H$ on $\Phi$, and from its value the mean force exerted on the root cap can readily be deduced by means of Eq. (16).

In the data represented in Fig. 10, $H$ takes three different values but $\langle d\rangle$ is the same in all samples. In order to further validate the scaling proposed in (16), we performed root growth simulations for several values of $R_{\min }$ and $R_{\max }$. In the simulations presented in the previous section, we had $\langle d\rangle=$ $3.1 \mathrm{~mm}$ for 1500 grains with radii varying between $R_{\min }=$ $1 \mathrm{~mm}$ and $R_{\max }=2 \mathrm{~mm}$. We prepared two more samples of mean particle diameters 1.6 and $4.2 \mathrm{~mm}$. In the first case we reduced $R_{\min }$ and increased the number of particles to 2000 to keep the same volume as the reference sample. In the second case, we increased $R_{\max }$ and reduced the number of particles to 1000 . The ratio $\lambda=R_{\max } / R_{\min }$ is 2 in the reference sample and, respectively, 2.5 and 5 in the two new samples.

Figure 11(a) shows the mean root-particle force $\langle F\rangle$ as a function of $K_{b}$ for the three samples. Here again, we see that the functional form (15) provides a nice fit to the data with hardness $H$ increasing considerably with $\langle d\rangle$. This is not surprising as for high bending stiffness the root dislodges nearly all particles on its path and thus the force $H$ directly reflects the contact forces, which for the stress induced by particle weights is proportional to $\langle d\rangle$ in $2 \mathrm{D}$. The increase of $\langle F\rangle$ is also related to the value of $\lambda$, which describes here the polydispersity of the samples. However, $H$ is not an increasing function of $\lambda$. This dependence needs to be determined by more simulations. Figure 11(b) displays the same data with normalized values of $\langle F\rangle$ and $K_{b}$. All the data collapse well and can again be fitted by the form (16) with the same value $a \simeq 0.15$ as before and shows once more that the scaling proposed here is robust with respect to the soil and root parameters.

We also checked by systematic simulations the influence of the gap parameter $s$ and the growth length $\ell_{g}$. The latter has little effect on the mean force $\langle F\rangle$ as far as its value is below $\langle d\rangle$. We find that the same scaling applies and the effect of $s$ is captured by the increase of $H$ with $s$. As for other parameters, more simulations are needed to determine the functional dependence of $H$ with respect to $s$.

Finally, we investigated the influence of cohesion on the root-particle force by assuming that the adhesion force $f_{n}^{c}$ in Eq. (2) is constant and localized at the contact point between the particles whereas the root-particle contacts are cohesionless. In the presence of cohesion, the root will have to overcome an additional tensile force $-f_{n}^{c}$ between the soil particles to advance [59]. For the simulations we used the sample of 1500 grains with particle diameter distribution between 2 and $4 \mathrm{~mm}$ and a mean diameter $\langle d\rangle=3.1 \mathrm{~mm}$
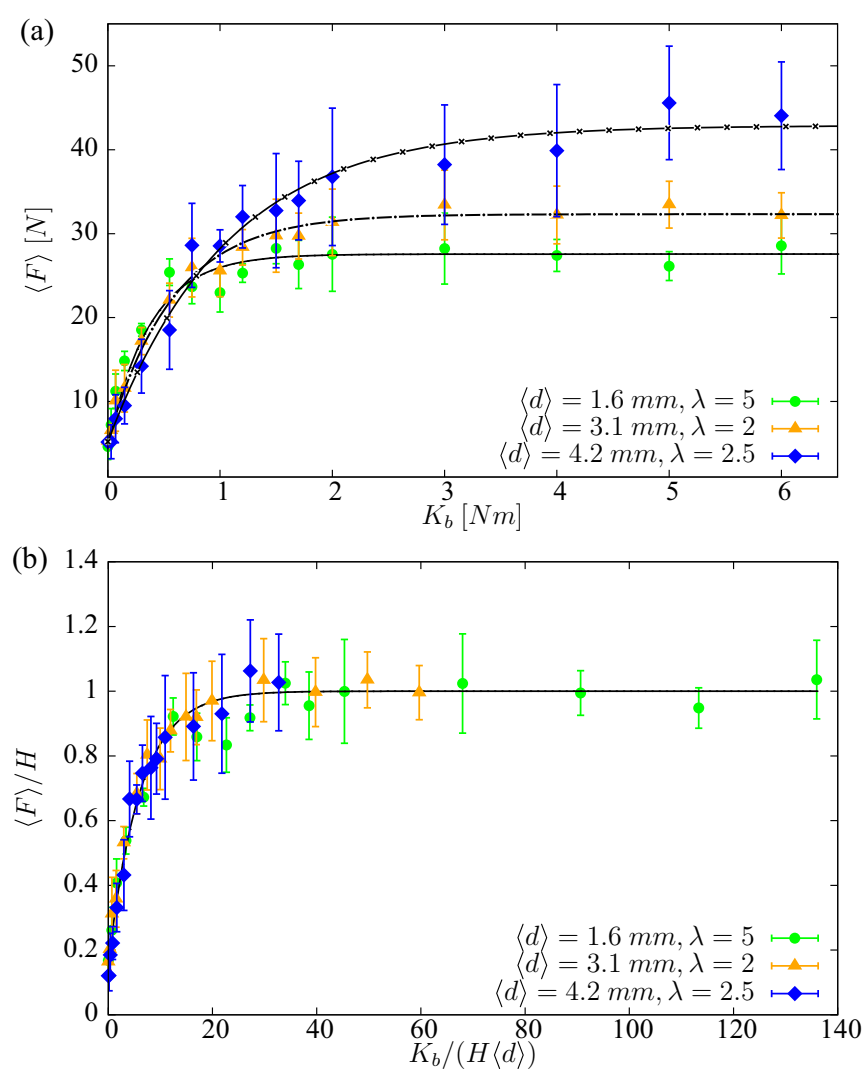

FIG. 11. (a) The mean force as a function of the root bending stiffness for three samples with different values $\langle d\rangle$ and size ratio $\lambda$. (b) The same data points with the mean force normalized by soil hardness $H$ and bending stiffness normalized by $H\langle d\rangle$. The lines are exponential fits according to Eq. (15) in (a) and Eq. (16) in (b).

with a root diameter larger than the gap between the particles $(s=1.54)$. We used three values of $f_{n}^{c}: 0,2\langle m\rangle g$, and $25\langle m\rangle g$. Figure 12(a) shows $\langle F\rangle$ as a function of $K_{b}$ for the three samples. The data follow an increasing exponential function as in cohesionless samples. The hardness $H$ has nearly the same value for $f_{n}^{c}=0$ and $f_{n}^{c}=2\langle m\rangle g$ but increases for $f_{n}^{c}=$ $25\langle m\rangle g$ as expected. The same data in normalized coordinates shown in Fig. 12(b) collapse on a curve well fit by Eq. (16).

As the cohesion due to capillary and clayey forces is a major ingredient of soils, this result considerably extends the relevance of Eq. (16) with its scaling parameters for the analysis of root-particle forces. In practice, the effects of cohesion and packing fraction can not be easily separated. In the simulations we considered the same sample and changed the value of adhesion force. But when a cohesive granular material is prepared by pouring the particles into a box, the packing fraction decreases as the cohesion increases, so that the combined effect of these parameters may cancel out. Such effects need to be investigated in order to determine how the hardness parameter $H$ depends on various soil parameters.

\section{CONCLUSIONS}

In this paper we introduced a simple discrete numerical model of root growth inside a granular soil. This model 

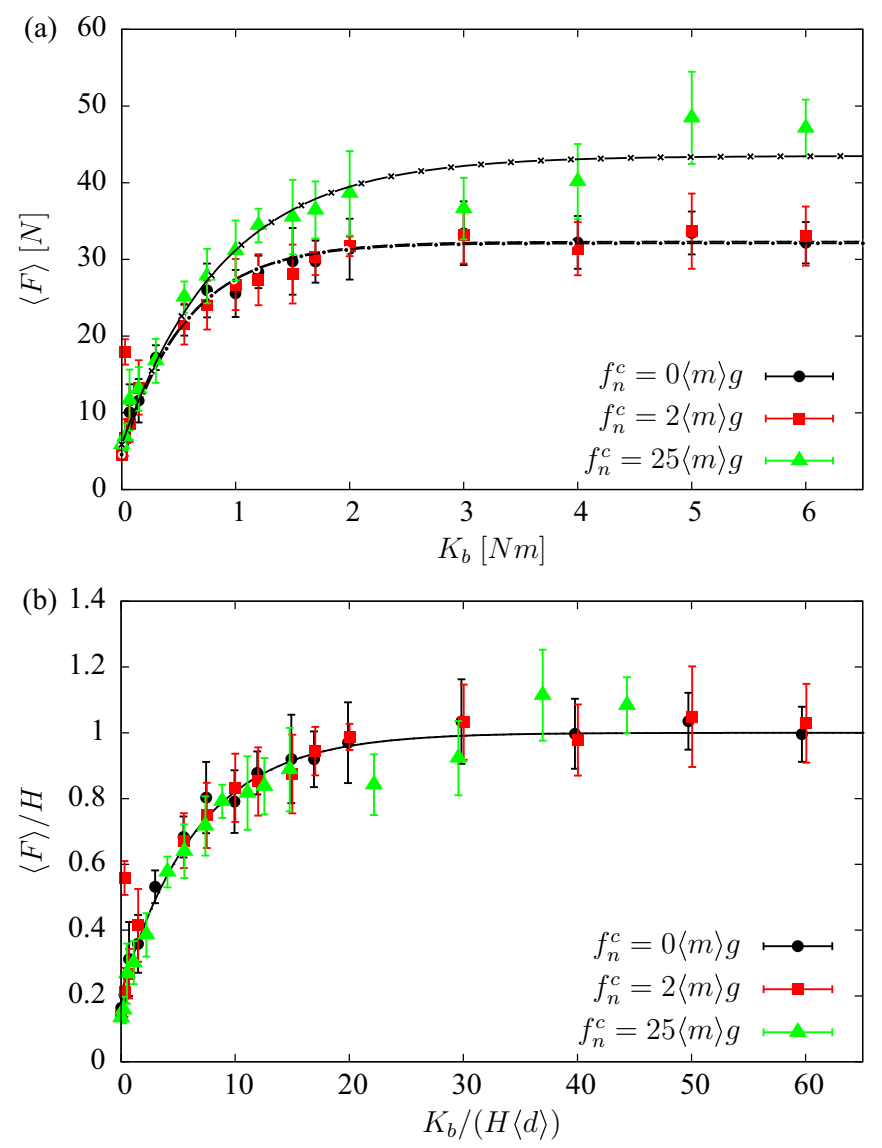

FIG. 12. (a) The mean force as a function of the bending stiffness for three samples with three values of the adhesion force: $0,2\langle m\rangle g$, and $25\langle m\rangle g$. The solid lines are exponential fits. (b) The same data with normalized coordinates. The solid line is the functional form of Eq. (16).

accounts for the root flexibility and its stiffening during its growth. At constant growth rate, the root development is guided by the disordered texture of the granular medium and the reaction forces exerted by the particles on the meristem, modeled here as the growing segment of the root. We found that these forces are well above the mean particle weight almost independently of the depth, and their statistical distribution reflects that of interparticle forces with an exponentially decaying number of strong forces. This feature is independent of the soil characteristics and root flexibility once the forces are normalized by the mean force.

By means of extensive simulations, we also showed that the mean root-particle force $F$ is an increasing exponential function of the root bending stiffness $K_{b}$ with an asymptotic value $F \rightarrow H$ that is a function of the soil characteristics such as packing fraction $\Phi$, mean particle size $\langle d\rangle$, and cohesion $f_{n}^{c}$. With a low bending stiffness, the root can change its direction in response to a weak contact force exerted by a particle, whereas with high bending stiffness the reorientation of the root requires larger contact forces that may dislodge the particle before the reorientation can occur. Hence, the largest force $H$ corresponds to a very stiff root such that it advances along a nearly straight line by dislodging all particles on its pathway. More interestingly, our simulations suggest that the functional dependence of $F / H$ on $K_{b} /(\langle d\rangle H)$ is almost universal, the effect of soil characteristics being fully captured by the value of $H$. A practical implication of this scaling is that the mean force experienced by the root cap during its growth can be estimated from the values of $H$ (measurable by means of penetrometric tests), mean particle size $\langle d\rangle$, and root bending stiffness $K_{b}$. The effect of soil parameters on the value of $H$ can be further quantified by root growth simulations using a fixed high value of $K_{b}$ but many more soil samples with varying packing fraction, size polydispersity, and cohesion should be prepared.

In this work, we neglected the feedback of the root-particle force on the growth rate, which was assumed to be constant. This approximation was necessary to focus on the interaction of the root tip with force network. But the growth rate varies with the environmental stimuli. At large-enough values of the mechanical force exerted by the particles on the root tip, the root growth can even stop. This feedback is a consequence of the internal mechanisms of the root growth based on the variations of turgor pressure and continuous cellular division at the root tip. However, the force experienced by the root tip during its growth is independent of its growth rate and it reflects only the path taken by the root through the soil. In other words, it depends simply on whether the root can exert the force necessary to dislodge the particles on its pathway. But the feedback on the growth rate can certainly influence the root shape, which results from the path taken by the root tip. In all cases, in simulations it is possible to calculate the work performed by the root on the soil particles and it would be interesting to see how this work depends on the root flexibility and soil characteristics. Our results on the root shape and work of root growth will be published elsewhere.

The extensive parametric investigations presented in this paper were made possible by the 2D geometry of the system, which requires much less computation time and memory than in 3D. The key point in the process of root growth is the interactions of the root cap with the force network through its action on the particles. The particles that fully or partially belong to the weak force network are easily dislodged. For this reason, the weak root-particle forces reflect weak interparticle forces. Over a distance of the order of the size of a "cage" of strong force chains (see Fig. 1), the root will have to dislodge particles sustaining strong forces. Hence, the strong root-particle forces arise from strong forces and larger length scales in the root morphology. This picture suggests that, as the distributions and morphologies of force networks are quite similar in $2 \mathrm{D}$ and $3 \mathrm{D}$, the root growth characteristics and forces should be similar, too. But we presently work on an equivalent 3D model of root growth for comparison with $2 \mathrm{D}$.

From the biological point of view, providing an analytical formulation of the reaction forces experienced by a root growing in a heterogenous medium is significant as this information is almost impossible to measure in the field. Linking this information to a model of root shape, which is easy to characterize by image analyses, will also give an indirect way to estimate the mechanical history of a growing root. This work will finally lay the groundwork to address the issues of mechanosensing and thigmomorphogenesis [60] in "the hidden part" of plants. 


\section{ACKNOWLEDGMENTS}

This work was carried out within the framework of the RoSoM project. This project is supported by Agropolis
Fondation under the reference ID 1202-073 through the Investissements d'avenir program (Labex Agro: ANR-10LABX-001-01).
[1] J. P. Lynch, Austr. J. Bot. 55, 493 (2007).

[2] A. Hodge, G. Berta, C. Doussan, F. Merchan, and M. Crespi, Plant Soil 321, 153 (2009).

[3] B. G. Forde, J. Exp. Bot. 60, 3989 (2009).

[4] L. Dupuy, P. J. Gregory, and A. G. Bengough, J. Exp. Bot. 61, 2131 (2010).

[5] A. G. Bengough, Plant Soil 360, 15 (2012).

[6] T. A. Valentine, P. D. Hallett, K. Binnie, M. W. Young, G. R. Squire, C. Hawes, and A. G. Bengough, Ann. Bot. 110, 259 (2012).

[7] M. D. Ho, B. C. McCannon, and J. P. Lynch, J. Theor. Biol. 226, 331 (2004).

[8] X. Draye, Y. Kim, G. Lobet, and M. Javaux, J. Exp. Bot. 61, 2145 (2010).

[9] C. Doussan, A. Pierret, E. Garrigues, and L. Pages, Plant Soil 283, 99 (2006).

[10] C. Doussan, L. Pages, and A. Pierret, Agronomie 23, 419 (2003).

[11] V. Clausnitzer and J. Hopmans, Plant Soil 164, 299 (1994).

[12] A. G. Bengough, M. F. Bransby, J. Hans, S. J. McKenna, T. J. Roberts, and T. A. Valentine, J. Exp. Bot. 57, 437 (2006).

[13] A. G. Bengough and J. M. Kirby, New Phytologist 142, 421 (1999).

[14] E. Kolb, C. Hartmann, and P. Genet, Plant Soil 360, 19 (2012).

[15] B. W. Eavis, L. F. Ratliff, and H. M. Taylor, Agron. J. 61, 640 (1969).

[16] I. De Smet, P. J. White, A. G. Bengough, L. Dupuy, B. Parizot, I. Casimiro, R. Heidstra, M. Laskowski, M. Lepetit, F. Hochholdinger et al., Plant Cell 24, 15 (2012).

[17] C. Bécel, G. Vercambre, and L. Pages, Plant Soil 353, 169 (2012).

[18] A. G. Bengough, B. M. McKenzie, P. D. Hallett, and T. A. Valentine, J. Exp. Bot. 62, 59 (2011).

[19] A. R. Dexter, Plant Soil 98, 303 (1987).

[20] J. M. Kirby and A. G. Bengough, Eur. J. Soil Sci. 53, 119 (2002).

[21] M. Yang, P. Défossez, F. Danjon, S. Dupont, and T. Fourcaud, Plant Soil 411, 275 (2017).

[22] S. Upadhyaya, U. Rosa, and D. Wulfsohn, Application of the finite element method in agricultural soil mechanics, in Advances in Soil Dynamics (ASAE, Washington, 2002), Vol. 2, Chap. 2.

[23] S. Materechera, A. Alston, J. Kirby, and A. Dexter, Plant Soil 144, 297 (1992).

[24] D. I. MacKenzie, J. D. Nichols, M. E. Seamans, and R. J. Gutiérrez, Ecology 90, 823 (2009).

[25] P. J. Gregory, Plant Roots: Growth, Activity and Interaction with Soils (Blackwell, Oxford, UK, 2006), Vol. 100, http://aob. oxfordjournals.org/content/100/1/151.short.

[26] W. Whalley and A. Dexter, Plant Soil 157, 313 (1993).

[27] S. R. Tracy, C. R. Black, J. A. Roberts, and S. J. Mooney, J. Sci. Food Agric. 91, 1528 (2011).

[28] L. Clark, W. Whalley, and P. Barraclough, Plant Soil 255, 93 (2003).

[29] A. V. Vollsnes, C. M. Futsaether, and A. G. Bengough, Eur. J. Soil Sci. 61, 926 (2010).
[30] L. H. Stolzy and K. P. Barley, Soil Sci. 105, 297 (1968).

[31] A. G. Bengough and C. E. Mullins, J. Soil Sci. 41, 341 (1990).

[32] A. G. Bengough and C. J. MacKenzie, J. Exp. Bot. 45, 95 (1994).

[33] W. Pfeffer, Druck- und Arbeitsleistung durch wachsende Pflanzen (S. Hirzel, Leipzig, 1983) http://www. biodiversitylibrary.org/item/47172.

[34] P. Kuzeja, P. Lintilhac, and C. Wei, J. Plant Physiol. 158, 673 (2001).

[35] R. Misra, A. Dexter, and A. Alston, Plant Soil 95, 315 (1986).

[36] F. Radjai, D. E. Wolf, M. Jean, and J. J. Moreau, Phys. Rev. Lett. 80, 61 (1998).

[37] F. Radjai, Comp. Rend. Phys. 16, 3 (2015).

[38] C. Liu, S. R. Nagel, D. A. Schecter, S. N. Coppersmith, S. Majumdar, O. Narayan, and T. A. Witten, Science 269, 513 (1995).

[39] F. Radjai, M. Jean, J.-J. Moreau, and S. Roux, Phys. Rev. Lett. 77, 274 (1996).

[40] C. Voivret, F. Radjai, J.-Y. Delenne, and M. S. El Youssoufi, Phys. Rev. Lett. 102, 178001 (2009).

[41] V. Richefeu, M. S. El Youssoufi, E. Azéma, and F. Radjai, Powder Technol. 190, 258 (2009).

[42] J. C. Petit and E. Medina, Phys. Rev. E 98, 022903 (2018).

[43] P. A. Cundall and O. D. L. Strack, Geotechnique 29, 47 (1979).

[44] F. Radjai and F. Dubois (eds.), Discrete Element Modeling of Granular Materials (Wiley, New York, 2011).

[45] C. Voivret, F. Radjai, and J.-Y. Delenne, in Discrete-Element Modeling of Granular Materials, edited by F. Radjai and F. Dubois (Wiley-ISTE, New York, 2011), pp. 123-151.

[46] J.-Y. Delenne, V. Richefeu, V. Topin, and F. Radjai, in Discrete Numerical Modeling of Granular Materials, edited by F. Radjai and F. Dubois (Wiley-ISTE, New York, 2011).

[47] J.-Y. Delenne, Ph.D. thesis, Université Montpellier II, Sciences et Techniques du Languedoc, 2002.

[48] J. Shäfer, S. Dippel, and D. E. Wolf, J. Phys. I France 6, 5 (1996).

[49] S. Dippel, G. G. Batrouni, and D. E. Wolf, Phys. Rev. E 56, 3645 (1997).

[50] C. Voivret, F. Radjaï, J.-Y. Delenne, and M. S. El Youssoufi, Phys. Rev. E 76, 021301 (2007).

[51] L. Guillon, M. El Mecherki, S. Altenburger, P. L. Graumann, and I. J. Schalk, Environ. Microbiol. 14, 1982 (2012).

[52] A. G. Bengough, C. Croser, and J. Pritchard, Plant Soil 189, 155 (1997).

[53] M. P. Allen and D. J. Tildesley, Computer Simulation of Liquids (Clarendon Press, New York, 1989).

[54] M. Meffeja, Ph.D. thesis, University Rennes 1, France, 2012.

[55] T. S. Majmudar and R. P. Behringer, Nature 435, 1079 (2005).

[56] F. Radjai, S. Roux, and J. J. Moreau, Chaos 9, 544 (1999).

[57] A. G. Bengough and C. E. Mullins, Plant Soil 41, 341 (1991).

[58] J.-C. Quezada, G. Saussine, P. Breul, and F. Radjai, Sci. Rep. 4, 5707 (2014).

[59] F. Radjai and V. Richefeu, Philos. Trans. R. Soc. A 367, 5123 (2009).

[60] J. Braam, New Phytol. 165, 373 (2005). 